\title{
Combined effects of Lenvatinib and iodine-131 on cell apoptosis in nasopharyngeal carcinoma through inducing endoplasmic reticulum stress
}

\author{
GUOYU WANG ${ }^{*}$, JUHUA ZHUANG* , JING NI, YING YE, SAIFEI HE and WEI XIA \\ Department of Nuclear Medicine, The Seventh People's Hospital of Shanghai University of \\ Traditional Chinese Medicine, Shanghai 200137, P.R. China
}

Received October 16, 2016; Accepted April 26, 2018

DOI: $10.3892 /$ etm.2018.6652

\begin{abstract}
Nasopharyngeal carcinoma (NPC) is a type of malignant tumor characterized by high invasiveness, metastatic potential and worldwide incidence among patients with head and neck cancer. It has previously been demonstrated that Lenvatinib (LEB) is an efficient anti-cancer agent by multi-targeting of tyrosine kinase inhibitors. Iodine-131 (I-131) therapy has been accepted for the treatment of thyroid cancer and other carcinomas. In the present study, the combined effects of LEB and I-131 were investigated on NPC and the potential signal pathway mediated by LEB and I-131 on NPC cells was explored. Inhibitory effects of LEB and I-131 for NPC cells growth were investigated via MTT assay. Migration and invasion of NPC cells was studied by aggression assays following incubation of LEB and I-131. Apoptosis of NPC cells and tissues were analyzed via flow cytometry and TUNEL assay, respectively. Apoptosis-related gene expression levels in NPC cells following treatment with LEB and I-131 were determined by western blotting. Endoplasmic reticulum (ER) stress in NPC cells were analyzed in NPC cells and tumor tissues. Immunohistochemistry was used to analyze the efficacy of LEB and I-131 in NPC-tumor bearing mice. The results demonstrated that combined treatment of LEB and I-131 significantly inhibited growth, apoptosis, migration and invasion of NPC cells compared with single agent therapy. Apoptosis-related gene expression levels of caspase-3 and caspase- 9 were upregulated by LEB and I-131, whereas B call lymphoma-2, and P53 were downregulated in NPC cells
\end{abstract}

Correspondence to: Professor Wei Xia, Department of Nuclear Medicine, The Seventh People's Hospital of Shanghai University of Traditional Chinese Medicine, 358 Datong Road, Gaoqiao Town, Pudong, Shanghai 200137, P.R. China

E-mail: xiaweimedicine@163.com

${ }^{*}$ Contributed equally

Key words: Lenvatinib, iodine-131, nasopharyngeal carcinoma, apoptosis, endoplasmic reticulum stress and tumor tissues. In addition, signal mechanism analysis demonstrated that combined treatment of LEB and I-131 promoted expression levels of activating transcription factor 6 , inositol-requiring protein 1 (IER1), protein kinase RNA-like endoplasmic reticulum kinase (RERK), and C/EBP homologous protein in NPC cells. Furthermore, combined treatment of LEB and I-131 markedly inhibited in vivo growth of NPC and further prolonged survival of experimental mice compared with single agent and control groups. Immunohistochemistry indicated that c-jun N-terminal kinase and Caspase-3 were increased in NPS tumor tissues in xenograft models treated with LEB and I-131. Apoptotic bodies were also increased in tumors treated by LEB and I-131. In conclusion, these findings indicate that combined treatment of LEB and I-131 may inhibit NPC growth and aggression through upregulation of ER stress, suggesting combined treatment of LEB and I-131 may be a potential therapeutic schedule for the treatment of NPC.

\section{Introduction}

Nasopharyngeal carcinoma (NPC) is an omnibus terminology for a class of head and neck malignant epithelial tumors, which is characterized by different histopathological appearances and etiopathogenesis of tumorigenesis (1). Although the incidence rate of NPC is rare in clinical settings, the mortality rate is higher in human cancer as it is highly aggressive, according to a clinical investigation (2). It has also been demonstrated that NPC initiates from the epithelial cells of the nasopharynx and is well known as leading to a high rate of regional lymph node metastasis and distant metastasis in patients (3-5). Therefore, strategies of inhibition of migration and invasion may be an efficient potential clinical regiment for NPC therapy.

Generally, radiotherapy has been regarded as a primary treatment of NPC that can temporarily control tumor growth (6). However, high-dose irradiation and/or chemical drugs exhibit high probability of toxicities and may even destroy the fragile immune system in patients with NPC, which in return leads to rapid growth and distant nasopharyngeal invasion (7-9). Iodine-131 (I-131) therapy has been accepted for the treatment of thyroid cancer due to lower radiation of $\beta$-radial and easy excretion. A number of reports have focused 
on I-131 therapy for the treatment of thyroid cancer $(10,11)$. The efficacy of I-131 has been previously investigated for fewer side effects for patients with cancer. Risse et al (12) have investigated the efficacy of I-131 on hepatocellular cancer, and results indicated that I-131-lipiodol exhibited the same efficacy but with fewer side effects compared other radioactive therapy procedures. Zhao et al (13) have also explored novel specific strategies of monoclonal antibody BAC5, an anti-nasopharyngeal cancer monoclonal antibody, conjugated with Chinese cobra and I-13 for NPC therapy. Efficacies of I-131 in radiotherapy have been investigated in clinical trials $(14,15)$. These studies indicated that I-131 combined with other anti-cancer therapy may contribute to neoplasm regression and metastasis suppression.

Lenvatinib (LEB) is a multi-targeted tyrosine kinase inhibitor targeting fibroblast growth factor receptor (FGFR)1-4, platelet-derived growth factor (PDGF) receptor (PDGFR)- $\beta$, Ret, vascular endothelial growth factor (VEGF) receptor (VEGFR)1-3 and KIT, which can bind with various receptors associated with tumor angiogenesis (16). Although anti-tumor activities of LEB against various human tumors have been reported in preclinical experiments and in clinical trials, the molecular mechanism of anti-angiogenesis mediated by LEB has not been elaborated in the progression of NPC (17-19). Previous research has indicated that LEB has clinical benefits in renal cancer cells by targeting VEGFR1-3, FGFR1-4, PDGFR- $\beta$, Ret, and KIT signaling pathways (20). In addition, several agents that target the VEGF pathway have shown clinical benefits in inhibition of metastatic tumors, which suggested that target receptors of VEGF in cancer cells serve a vital role in tumor therapy $(21,22)$. Furthermore, clinical research has demonstrated that LEB combined with everolimus significantly extends overall survival compared with either LEB or everolimus alone in patients with metastatic renal cancer cells (23).

In the present study, the combined effects of LEB and I-131 were investigated on NPC cells both in vitro and in vivo. Preliminary results of the combined effects of LEB and I-131 on HK-1 cells are presented. The present findings suggest that combination of LEB and I-131 significantly inhibits growth and aggressiveness of NPC through upregulation of endoplasmic reticulum (ER) stress. Notably, apoptosis of NPC cells was markedly promoted via combined management of LEB and I-131.

\section{Materials and methods}

Ethics statement. The present study was approved by the Ethics Committee of the Seventh People's Hospital of Shanghai University of Traditional Chinese Medicine (Shanghai, China).

Cell culture and regents. NPC cell line HK-1 was purchased from the American Type Culture Collection (Manassas, VA, USA). HK-1 cells were cultured in Dulbecco's modified Eagle's medium (DMEM; Sigma-Aldrich; Merck KGaA, Darmstadt, Germany) supplemented with $10 \%$ fetal bovine serum (FBS; Gibco; Thermo Fisher Scientific, Inc., Waltham, MA, USA). All cells were cultured in a $37^{\circ} \mathrm{C}$ humidified atmosphere containing $5 \% \mathrm{CO}_{2}$.
MTT assay. HK-1 cells were incubated with Lenvatinib (5 mg/ml; Sigma-Aldrich; Merck KGaA) or/and I-131 (3 mg/ml; Zhengzhou Double-radioisotope Application Technology Co., Ltd., Zhengzhou, China) in 96-well plates for $48 \mathrm{~h}$ in triplicate for each condition. Following incubation, $20 \mu \mathrm{MTT}(5 \mathrm{mg} / \mathrm{ml})$ in PBS solution was added to each well and was further incubated for $4 \mathrm{~h}$. the majority of the medium was removed and $100 \mu 1$ dimethyl sulfoxide was added to each wells to dissolve the crystals. The optical density was measured at a wavelength of $450 \mathrm{~nm}$.

Cell invasion and migration assays. Cultured HK-1 cells were suspended as a density of $1 \times 10^{6}$ cells in $100 \mu \mathrm{l}$ serum-free DMEM medium for $24 \mathrm{~h}$ at $37^{\circ} \mathrm{C}$. HK- 1 cells were treated with Lenvatinib (5 mg/ml) or/and I-131 (3 mg/ml), with non-treated cells as a control, and then placed in the upper wells of BD BioCoat Matrigel Chambers (BD Biosciences, Franklin Lakes, NJ, USA) according to the manufacturer's instructions. RPMI-1640 (Thermo Fisher Scientific, Inc.) containing $20 \%$ FBS was used in the lower wells. Cells were incubated for $12 \mathrm{~h}$ at $37^{\circ} \mathrm{C}$. Cells were then stained with $0.5 \%$ crystal violet at room temperature for $18 \mathrm{~min}$. HK-1 cell migration and invasion were determined in at least three random fields (magnification, x20) under light microscopy for each sample. The extent of migration and invasion of HK-1 cells was mapped and quantified using Image J $1.37 \mathrm{v}$ (National Institutes of Health, Bethesda, MD, USA).

Animal study. A total of 30 male C57BL/6 nude mice (age, 6 weeks; weight, 25-30 g) were purchased from Shanghai SLAC Laboratory Animal Co., Ltd. (Shanghai, China). All animals were housed under pathogen-free conditions at room temperature with $50 \%$ humidity and 12-h light/dark cycle, with free access to food and water. A total of $200 \mu \mathrm{l} \mathrm{HK}-1$ cells $\left(1 \times 10^{6}\right)$ were injected into the backside of C57BL/6 nude mice. At 6 days following this administration, tumor-bearing mice were divided into four groups ( $\mathrm{n}=30 /$ group) and received treatment of PBS $(5 \mathrm{mg} / \mathrm{ml})$, or LEB $(5 \mathrm{mg} / \mathrm{kg})$ or/and I-131 (3 $\mathrm{mg} / \mathrm{kg}$ ). Treatments were administered once daily for 10 days. Tumor diameters were recorded every 2 days. Tumor volume was calculated according to the following formula: $\mathrm{V}=(\mathrm{LxWxW}) / 2$, where $\mathrm{V}$ is tumor volume, $\mathrm{W}$ is tumor width, $\mathrm{L}$ is tumor length. The experimental mice were sacrificed when tumors reached a length of $18 \mathrm{~mm}$.

Western blot analysis. SDS-PAGE assays were used to analyze the affect of Lenvatinib or/and I-131 on protein expression in NPC cells. HK-1 cells were treated with Lenvatinib $(5 \mathrm{mg} / \mathrm{ml})$ or/and I-131 (3 mg/ml) for $48 \mathrm{~h}$, with non-treated cells as controls. HK-1 cells were harvested and homogenized at $4^{\circ} \mathrm{C}$ for $10 \mathrm{~min}$. Radioimmunoprecipitation assay solution (Thermo Fisher Scientific, Inc.) was used to extract protein and concentrations were measured by bicinchoninic acid assay. Proteins $(20 \mu \mathrm{g})$ were separated on $10 \%$ SDS-PAGE gels, followed by transfer to a polyvinylidene difluoride membrane. Membranes were incubated with skimmed milk (5\%) for $2 \mathrm{~h}$ at room temperature. Membranes were incubated with primary antibodies, including c-jun $\mathrm{N}$-terminal kinase (JNK; 1:1,200, ab112501), P38 (1:1,200; ab31828), activating transcription factor (ATF)6 (1:1,200; ab37149), inositol-requiring 
protein (IER)1 (1:1,200; ab37037; all Abcam, Cambridge, UK), protein kinase RNA-like endoplasmic reticulum kinase (RERK; 1:1,500, MA5-15705; Invitrogen; Thermo Fisher Scientific., Inc.), B cell lymphoma 2 (Bcl-2; 1:1,200; ab196495), P53 (1:1,200; ab131442), VEGF (1:1,200, ab69479), PDGF (1:1,200; ab32570), caspase-3 (1:1,200; ab13585), caspase- 9 (1:1,200; ab32539), metastasis-associated protein (MTA-1; 1:1,200; ab71153), fibronectin (FIB; 1:1,200; ab3413), C/EBP homologous protein (CHOP; 1:1,200; ab11419) and $\beta$-actin $\left(1: 1,200\right.$; ab6276; all Abcam) for $2 \mathrm{~h}$ at $37^{\circ} \mathrm{C}$. Samples were then incubated with rabbit anti-mouse IgG-horse radish peroxidase-conjugated secondary antibody $(1: 1,200$; sc-358914; Santa Cruz Biotechnology, Inc., Dallas, TX, USA) at room temperature for $2 \mathrm{~h}$ and protein expression was analyzed using a Benchmark automated staining system (Ventana Medical Systems, Inc., Tucson, AZ, USA). Data were analyzed using ImageJ 1.37v (National Institutes of Health).

Immunofluorescence. HK-1 cells were treated with Lenvatinib $(5 \mathrm{mg} / \mathrm{ml})$ or/and $\mathrm{I}-131(3 \mathrm{mg} / \mathrm{ml})$ for $48 \mathrm{~h}$ at $37^{\circ} \mathrm{C}$ and fixed with $10 \%$ formaldehyde in 6 -well plates for $30 \mathrm{~min}$ at $37^{\circ} \mathrm{C}$. Degree of ER injury in HK-1 cells was observed via scanning electron microscopy (gold sputter coating; magnification, x100). Additionally, HK-1 cells were incubated with DAPI for $2 \mathrm{~h}$ at $37^{\circ} \mathrm{C}$ and then incubated with mouse anti-human glucose-regulated protein 78 (GRP78) antibody (1:1,200; 119-14212; RayBiotech, Inc., Norcross, GA, USA) for $2 \mathrm{~h}$ at $37^{\circ} \mathrm{C}$, followed by incubation with green-fluorescent Invitrogen Alexa Fluor 488 goat anti-mouse IgG1 antibody (1:1,200; A-21121; Thermo Fisher Scientific, Inc.) at room temperature for $1 \mathrm{~h}$ to analyze the effects of Lenvatinib or/and I-131 on GRP78 expression. A Benchmark automated staining system was used for observation of purpose protein. Results were observed under light microscope (magnification, x20).

Immunohistochemistry. Tumors sections were obtained from NPC xenograph mice and were fixed with $10 \%$ formaldehyde at $4^{\circ} \mathrm{C}$ for $8 \mathrm{~h}$ and embedded in paraffin. Tumor samples were cut into sections $(6 \mathrm{~mm})$ and antigen retrieval was also performed by incubating tissue sections at $93^{\circ} \mathrm{C}$ for $20 \mathrm{~min}$, followed by incubation on ice for $40 \mathrm{~min}$. Dehydration was performed by passing a graded concentrations of ethanol $(100,95,85,70$ and 50\%) Tumor sections were incubated with rabbit anti-human JNK (1:1,200; ab112501), caspase-3 (1:1,200; ab13847), hypoxia-inducible factor (HIF) $1 \alpha$ (1:1,200; ab51608), tumor necrosis factor (TNF)- $\alpha(1: 1,200$; ab6671) and VEGF $(1: 1,200$; ab46154; all Abcam) antibodies for $2 \mathrm{~h}$ at $37^{\circ} \mathrm{C}$, followed by incubation with anti-rabbit IgG-HRP secondary antibody (1:1,000; MBS435036; MyBioSource, San Diego, CA, USA) at room temperature for $1 \mathrm{~h}$ and washing with PBS. VENTANA System Software 12.3 (BenchMark, San Francisco, CA, USA) was used for observation of proteins.

Apoptosis assay. Flow cytometry and terminal deoxynucleotidyl-transferase-mediated dUTP nick end labeling (TUNEL) assays were used to analyze the apoptosis rate of HK-1 cells and apoptotic bodies in tumor tissues. Flow cytometry analyzed apoptosis rate of HK-1 cells via Annexin V-fluorescein isothiocyanate and propidium iodide staining (Annexin V-FITC kit; BD Biosciences). TUNEL assays were used to analyze the number of apoptosis body in tumors isolated form experimental mice through flow cytometry. All procedures were performed as detailed previously (24). Data were analyzed using FlowJo ${ }^{\mathrm{TM}}$ software v10.2 (FlowJo LLC, Ashland, OR, USA).

Histopathological examination using hematoxylin and eosin $(H \& E)$ staining. Tissues were fixed with $4 \%$ formaldehyde overnight at $4^{\circ} \mathrm{C}$. Tissues were embedded in paraffin and cut into 6-mm slices. Hematoxylin staining was performed for $8 \mathrm{~min}$ at room temperature and counterstain in eosin-phloxine solution was performed for $1 \mathrm{~min}$ at room temperature. After staining tissues were observed under an optical microscope (magnification, x200; Nikon Eclipse TE2000-U; Nikon Corporation, Tokyo, Japan) for histopathological analysis.

Statistical analysis. All data were presented as the means \pm standard error of the mean of three independent experiments. Data comparisons between two groups were performed using Student's t-test and comparisons of data between multiple groups were evaluated via one-way analysis of variance followed by Tukey's test. $\mathrm{P}<0.05$ was considered to indicate a statistically significant difference.

\section{Results}

Combined effects of LEB and I-131 on growth and aggressiveness of NPC cells in vitro. As presented in Fig. 1A, results indicated that combined treatment of LEB $(5 \mathrm{mg} / \mathrm{ml})$ and I-131 ( $3 \mathrm{mg} / \mathrm{ml}$ ) significantly inhibited growth of HK-1 NPC cells compared with either LEB or I-131. Migration and invasion assays demonstrated that combination of LEB $(10 \mathrm{mg} / \mathrm{ml})$ and I-131 $(10 \mathrm{mg} / \mathrm{ml})$ significantly suppressed migration and invasion of HK-1 cells following incubation for $48 \mathrm{~h}$ (Fig. 1B and $\mathrm{C}$ ). Western blot analysis also demonstrated that tumor metastasis-related genes MTA-1 and FIB were significantly decreased by combined treatment of LEB $(5 \mathrm{mg} / \mathrm{ml})$ and I-131 (3 mg/ml) compared with either agent alone (Fig. 1D). These data indicate that combined treatment of LEB and I-131 significantly inhibits growth and aggressiveness of NPC cells in vitro.

Combined effects of LEB and I-131 on apoptosis of NPC cells and tumor angiogenesis-related protein in vitro. The effects of LEB and I-131 on apoptosis of NPC cells and tumor angiogenic protein expression levels were analyzed in vitro. It was observed that combined treatment of LEB $(5 \mathrm{mg} / \mathrm{ml})$ and I-131 $(3 \mathrm{mg} / \mathrm{ml})$ significantly promoted apoptosis of HK-1 cells compared with single agents (Fig. 2A). Pro-apoptosis gene expression levels of Caspase- 3 and Caspase-9 were significantly upregulated by LEB and I-131 in HK-1 cells (Fig. 2B). Anti-apoptosis gene expression levels of $\mathrm{Bcl}-2$ and P53 were significantly downregulated by combined treatment with LEB and I-131 in NPC cells (Fig. 2C). Furthermore, it was demonstrated that combined treatment with LEB $(3 \mathrm{mg} / \mathrm{ml})$ and $\mathrm{I}-131(3 \mathrm{mg} / \mathrm{ml})$ significantly inhibited tumor angiogenesis-related protein expression including VEGF and PDGF in HK-1 cells (Fig. 2D). These results suggest that combined treatment with LEB and I-131 may significantly 
A

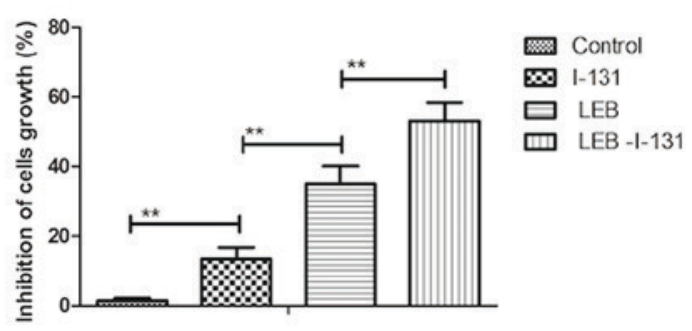

C
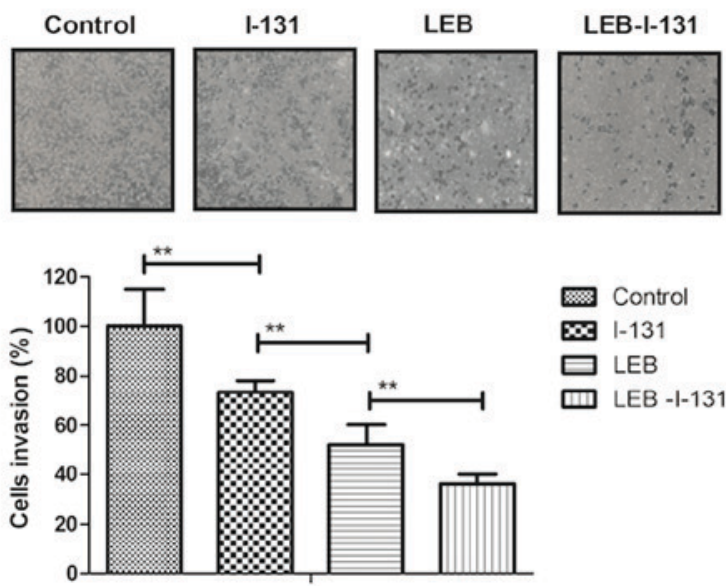

B

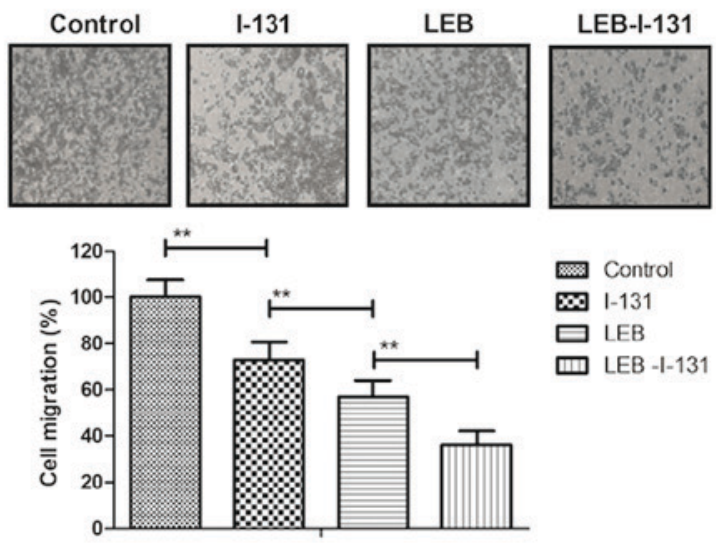

D

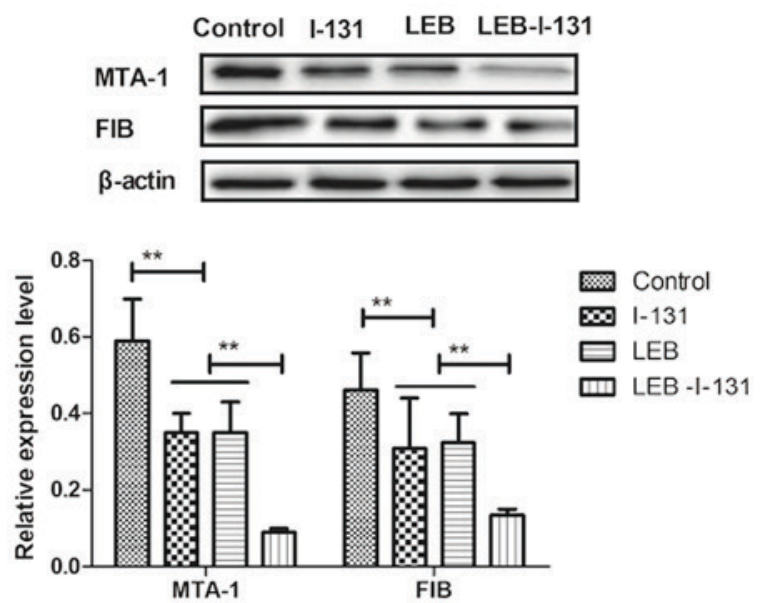

Figure 1. Combination of LEB and I-131 inhibits growth and aggressiveness of nasopharyngeal carcinoma cells in vitro. (A) Growth of HK-1 cells following treatment with LEB or/and I-131 determined by MTT assay. (B) Migration (magnification, x20) and (C) invasion (magnification, x20) of HK-1 cells following treatment with LEB or/and I-131 determined via aggression assays. (D) Expression levels of MTA-1 and FIB in HK-1 cells following treatment with LEB or/and I-131 determined by western blotting. The data are presented as the mean + standard error of the mean of three independent experiments. "* $\mathrm{P}<0.01$. LEB, Lenvatinib; I-131, iodine-131; MTA-1, metastasis-associated protein; FIB, fibrinogen.

promote apoptosis and inhibit tumor angiogenesis-related protein of NPC cells in vitro.

Combination of LEB and I-131 regulates apoptosis of NPC cells through ER stress. In order to elucidate the mechanism of NPC cells mediated by LEB and I-131, changes of ER stress in HK-1 cells were analyzed. ATF6, IER1, RERK and CHOP expression levels were analyzed in HK-1 cells. The results demonstrated that combined treatment of LEB and I-131 upregulated ATF6, IER1 RERK and CHOP expression levels in HK-1 cells (Fig. 3A). It was also demonstrated that mRNA expression levels were significantly upregulated by combined treatment with LEB and I-131 in HK-1 cells (Fig. 3B). Immunolocalization indicated that structure of ER was damaged by combined treatment of LEB and I-131, as presented by the broken ER structure visible in Fig. 3C. In addition, results demonstrated that GRP78 expression was higher in HK-1 cells following combined treatment of LEB and I-131 (Fig. 3D). It was also demonstrated that numbers of adhered HK-1 cells were decreased by combined treatment of LEB and I-131 (Fig. 3E). Furthermore, JNK and P38 expression levels in HK-1 cells were also upregulated by combined treatment of LEB and I-131 (Fig. 3F). Collectively, these results suggest that combined treatment of LEB and I-131 can promote apoptosis of NPC cells through increasing ER stress.

Combined treatment of LEB and I-131 inhibits tumor growth and promotes apoptosis in xenograft mice model. The in vivo effects of combined treatment of LEB and I-131 were analyzed in a xenograft mice model. As presented in Fig. 4A, tumor growth was significantly inhibited by combined treatment with LEB and I-131 in a 30-day observation. TUNEL assay revealed that apoptotic bodies were increased by combined treatment of LEB and I-131 in tumors in experimental xenograft mice (Fig. 4B). Immunohistochemistry demonstrated that JNK and Caspase-3 expression levels were upregulated in NPC tumors in mice treated with LEB and I-131 (Fig. 4C). In addition, TNF- $\alpha$ and HIF-1 $\alpha$ expression levels were also upregulated by combined treatment of LEB and I-131 in tumors in experimental xenograft mice (Fig. 4D). Furthermore, H\&E staining assay demonstrated that angiogenesis and VEGF expression levels were significantly decreased in tumors following treatment with LEB and I-131 compared with single agent and PBS groups (Fig. 4E). Collectively, these results indicate that combined treatment of LEB and I-131 inhibits tumor growth and promotes apoptosis in a xenograft mice model. 


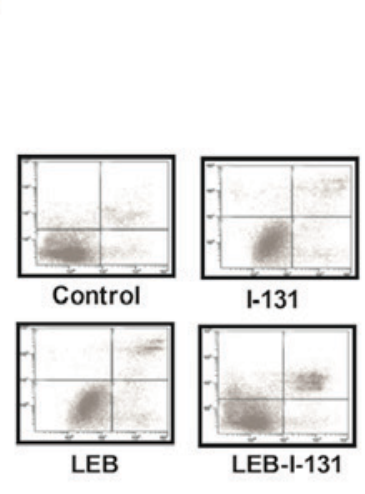

C
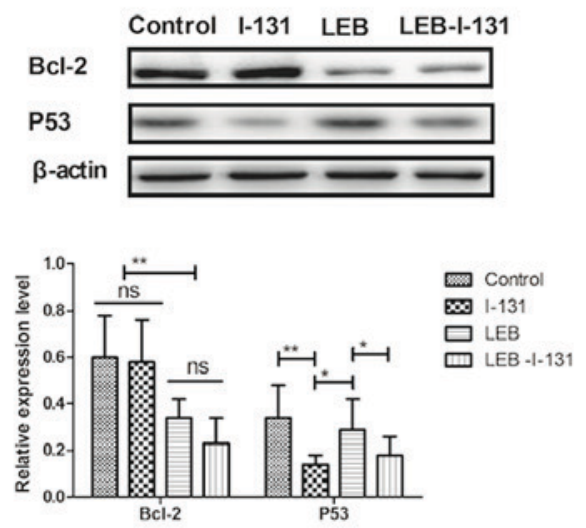
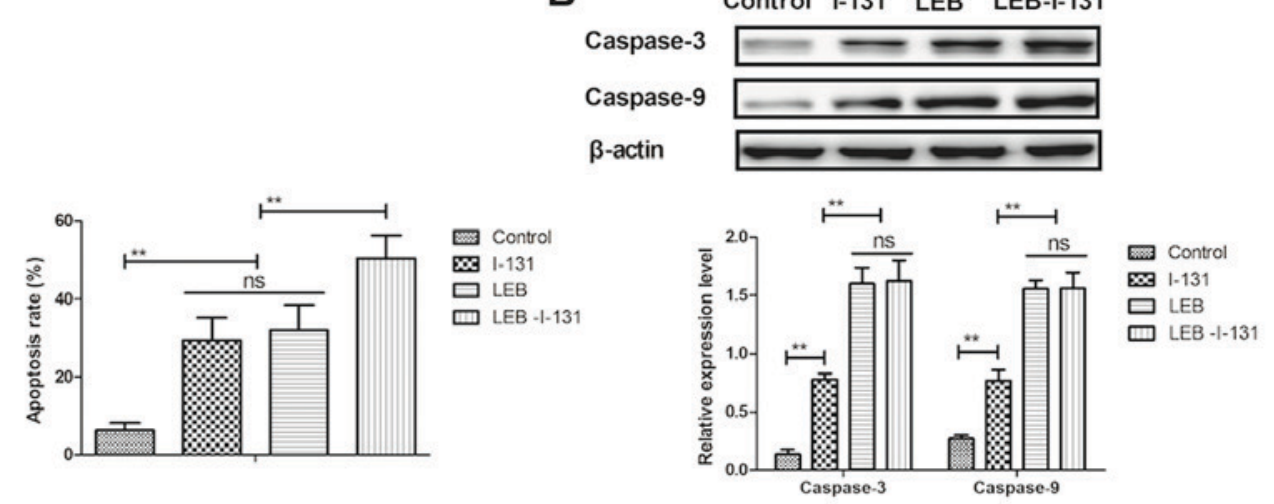

D
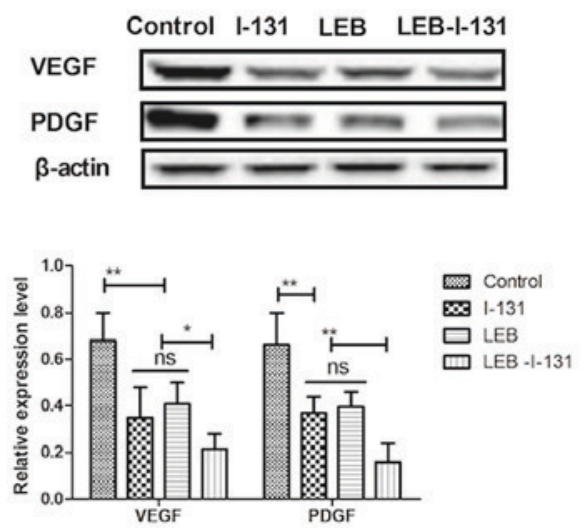

Figure 2. Combination of LEB and I-131 promotes apoptosis of nasopharyngeal carcinoma cells and inhibits tumor angiogenesis-related protein in vitro. (A) Apoptosis rate of HK-1 cells following treatment with LEB or/and I-131 determined by flow cytometry. (B) Protein expression levels of Caspase-3 and Caspase-9 in HK-1 cells determined by western blotting. (C) Protein expression levels of Bcl-2 and P53 in HK-1 cells determined by western blotting. (D) VEGF and PDGF expression levels in HK-1 cells determined by western blotting. The data are presented as the mean + standard error of the mean of three independent experiments. ${ }^{*} \mathrm{P}<0.05,{ }^{* *} \mathrm{P}<0.01$. LEB, Lenvatinib; I-131, iodine-131; Bcl-2, B cell lymphoma 2; VEGF, vascular endothelial growth factor; PDGF, platelet-derived growth factor.

\section{Discussion}

NPC is a rare, highly aggressive cancer (25). Standard therapies for NPC remain toxic and ineffective (26). I-131 is known as producing relatively low radiation, and has exhibited anti-cancer effects in several types of human cancer, including thyroid and hepatocellular cancer (10-12). In addition, targeted therapy of LEB has presented the potential to completely eradicate tumors, but with low toxicity to normal human tissues during cancer treatment $(27,28)$. In the present study, the combined effects of I-131 and LEB for the treatment of NPC were investigated in vitro and in vivo. The findings demonstrated that combined treatment of I-131 and LEB not only significantly inhibited growth, apoptosis, and aggressiveness of NPC cells in vitro, but also suppressed NPC cells growth in xenograft mice in vivo. Notably, these findings indicated that combined treatment of I-131 and LEB promoted apoptosis of NPC cells through upregulation of ER stress.

Currently, inhibition of tumor growth is the primary and most fundamental facet in the progression of cancer treatment (29). Enhancement of apoptosis in tumor tissues has been reported to contribute to inhibition of tumor growth and may lead to immunological recognition of immune system in cancer patients (30). Conventional radiotherapy is the most frequently used treatment of NPC, however, radiotherapy injury seriously may damage the immune system (31). I-131 radioimmunotherapy has proven advantageous in the treatment of human thyroid cancer due to lower radiation, easier excretion and higher accuracy (32).

Notably, the role of I-131 in theranostic action has been identified in tumor diagnostics $(33,34)$. Ionizing effects of on I-131 target radiotherapy on cancer cells have been identified in rabbit VX2 tumor models (35). In the present study, the efficacy of I-131 was explored in NPC cells and xenograft mice. The results have indicated that I-131 inhibits growth and aggressiveness of NPC cells. I-131 induces apoptosis of NPC cells through ER stress. However, it was also observed that the efficacy of single I-131 treatment was limited and yielded unsatisfactory effects.

LEB is an oral multi-targeted tyrosine kinase inhibitor of VEGFR1-3, FGFR1-4, PDGFR- $\beta$, Ret, and KIT. Previous research has indicated that VEGF, FGFR1-4, PDGFR- $\beta$, Ret, and KIT-mediated angiogenesis is a key factor in the processes of tumor angiogenesis (36). LEB is a target-therapeutic drug due to its role as an efficient anticancer agent in patients with advanced solid tumor (37). In addition, Dubbelman et al (38) have recently explored the efficacy and pharmacokinetics of LEB in patients with advanced solid tumors or lymphomas. 
A

Control I-131 LEB LEB-I-131

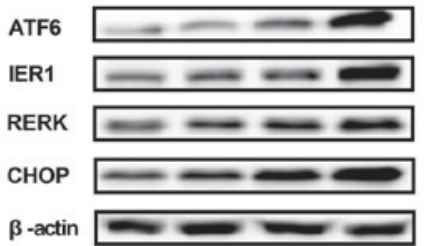

D

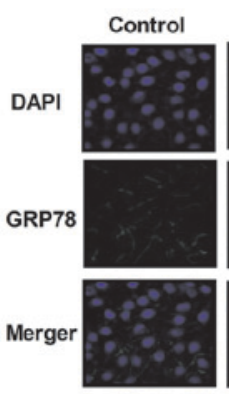

B

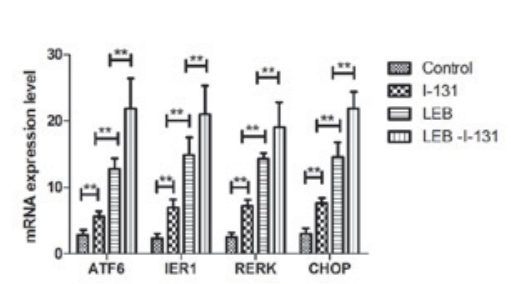

E

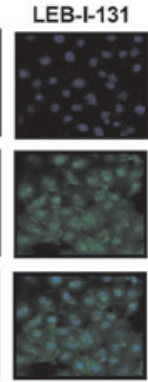

C

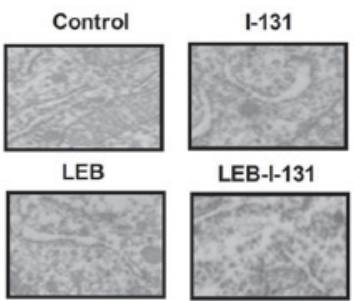

$\mathbf{F}$

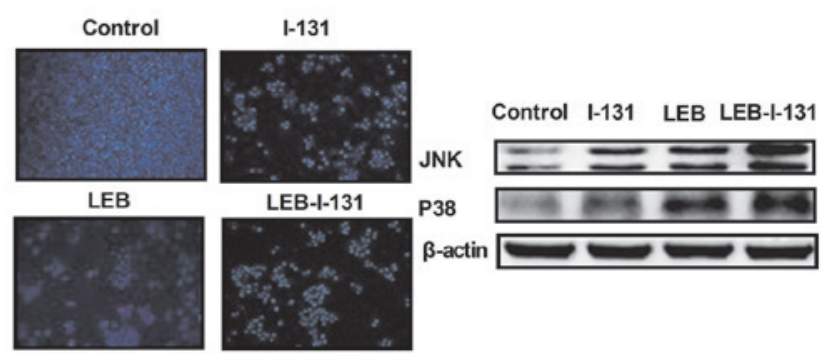

Figure 3. Combination of LEB and I-131 regulates apoptosis of nasopharyngeal carcinoma cells through ER stress. (A) ATF6, IER1, RERK and CHOP expression levels in HK-1 cells following treatment with LEB or/and I-131 determined by western blotting. (B) Analysis of mRNA expression levels of ATF6, IER1, RERK and CHOP expression levels in HK-1 cells. (C) Effects of LEB or/and I-131 on ER in HK-1 cells (magnification, x100). (D) GRP78 expression in HK-1 cells following combined treatment of LEB and I-131 determined by immunofluorescence (magnification, x20). (E) Adhered morphology of HK-1 cells following treatment with LEB or/and I-131 (magnification, x20). (F) JNK and P38 expression levels in HK-1 following treatment with LEB or/and I-131. The data are presented as the mean + standard error of the mean of three independent experiments. ${ }^{* *} \mathrm{P}<0.01$. LEB, Lenvatinib; I-131, iodine-131; ATF6, activating transcription factor 6; IER1, inositol-requiring protein 1; RERK, protein kinase RNA-like endoplasmic reticulum kinase; $\mathrm{CHOP}$, C/EBP homologous protein; GRP78, glucose-regulated protein 78; JNK, c-jun N-terminal kinase.

A

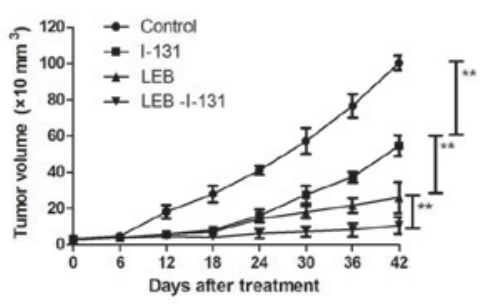

C

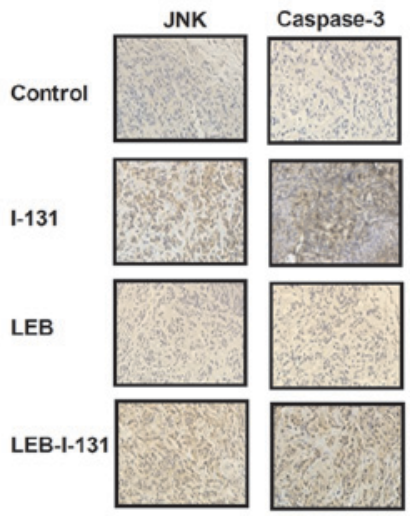

B

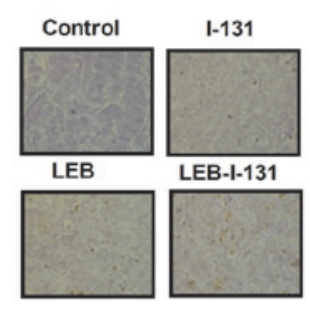

E

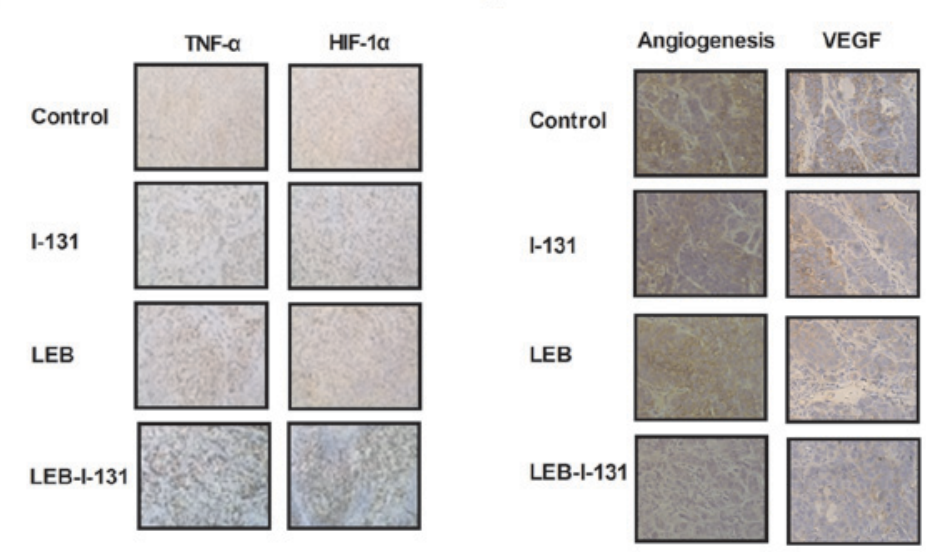

Figure 4. In vivo effects of combined treatment of LEB and I-131 in an NPC xenograft mice model. (A) Effects of combined treatment of LEB and I-131 on tumor growth in NPC xenograft mice model. (B) Apoptotic bodies in tumors following treatment with LEB or/and I-131 determined by terminal deoxynucleotidyl-transferase-mediated dUTP nick end labeling assay (magnification, x20). (C) JNK and Caspase-3 expression levels in tumors following treatment with LEB or/and I-131 determined by immunohistochemistry (magnification, x20). (D) Expression levels of TNF- $\alpha$ and HIF-1 $\alpha$ in tumors following treatment with LEB or/and I-131 determined by immunohistochemistry (magnification, x20). (E) Angiogenesis and VEGF expression in tumors following treatment with LEB or/and I-131 determined by hematoxylin and eosin staining (magnification, $\mathrm{x} 20$ ). ${ }^{* *} \mathrm{P}<0.01$. LEB, Lenvatinib; I-131, iodine-131; NPC, nasopharyngeal carcinoma; JNK, c-jun N-terminal kinase; TNF- $\alpha$, tumor necrosis factor- $\alpha$; HIF-1 $\alpha$, hypoxia-inducible factor-1 $\alpha$; VEGF, vascular endothelial growth factor. 
Tohyama et al (39) have also indicated the anti-tumor activity of LEB by targeting multiple tyrosine kinase receptors in preclinical human thyroid cancer models. In the present study, the combined efficacy of I-131 and LEB for the treatment of NPC was reported. The present findings indicate that combined treatment of I-131 and LEB significantly suppresses growth and aggression of NPC both in vitro and in vivo. Notably, the findings also indicated that apoptosis of NPC was enhanced by synergetic treatment of I-131 and LEB mediated by ER stress.

Apoptosis resistance is the most important characteristic of NPC, which limits the efficacy of anti-cancer drugs in clinical settings (40). It has been identified that upregulation of ER stress may induce apoptosis of cancer cells $(41,42)$. ER stress upregulation potentiating apoptosis also has been observed in human triple negative breast carcinoma cells (43). In the present study, it has been demonstrated that ATF6, IER1 RERK, and CHOP expression levels were increased by synergetic treatment of I-131 and LEB in NPC cells and tumor tissues. These molecules contribute to ER stress upregulation and promote apoptosis of PC cells and tumor tissues.

In conclusion, the present study has demonstrated the inhibitory effects of I-131 and LEB in NPC cells and an NPC xenograft mice model. The combination of target therapy of LEB and lower-radiation I-131 for NPC therapy was investigated. Notably, the present data indicates that combined treatment of I-131 and LEB significantly inhibits the tumor growth in NPC xenograft mice. The findings also suggested that the combined treatment of I-131 and LEB has potential application for the treatment of patients with NPC. However, further investigations are required to identify the pharmacodynamics and toxicology of combined treatment of I-131 and LEB. Clinical experiments may be considered to confirm the conclusions of the present study.

\section{Acknowledgements}

Not applicable.

\section{Funding}

The present study was supported by the Key Discipline Construction Project of Pudong Health Bureau of Shanghai (grant no. PWZxk2017-06), the Natural Science Foundation of China (grant no. 81571718), the Science and Technology Development Fund of Shanghai Pudong New Area (rant no. PKJ2016-Y19), the Budgetary Fund of Shanghai University of TCM (grant no. 2015YSN59) and the Talents Training Program of Seventh People's Hospital of Shanghai University of TCM (grant nos. QMX2017-01 and XX2017-06).

\section{Availability of data and materials}

The datasets used and/or analyzed during the present study are available from the corresponding author on reasonable request.

\section{Authors' contributions}

GW, JZ and WX conceived and designed the study. GW and JZ performed experiments and analyzed data. JN, YY and SH interpreted the data. WX drafted the manuscript. All authors read and approved the final manuscript.

\section{Ethics approval and consent to participate}

The present study was approved by the Ethics Committee of the Seventh People's Hospital of Shanghai University of Traditional Chinese Medicine.

\section{Patient consent for publication}

Not applicable.

\section{Competing interests}

The authors declare that they have no competing interests.

\section{References}

1. Ao R, Fu R, Dong D, Zhu X, Liu H and Xie K: Discerning primary tumors from metastases in synchronous nasopharyngeal squamous cell carcinoma and cutaneous squamous cell carcinoma: A case report and review of the literature. Oncol Lett 7: 1391-1394, 2014

2. Ceballos Saenz C, Argyris PP, Manivel JC, Urias Barreras CM and Koutlas IG: Nasopharyngeal hyalinizing clear cell carcinoma: Report of the histopathologic features of a case showing EWSR1 rearrangements by FISH and literature review. Int J Surg Pathol 22: 667-672, 2014.

3. Petersson F: Nasopharyngeal carcinoma: A review. Semin Diagn Pathol 32: 54-73, 2015.

4. Ren K, Wang W, Ma X, Guo F, Li P and Liu L: Nasopharyngeal carcinoma mimicking Aspergillosis rhinosinusitis: An unusual case report and review of the literature. Int J Clin Exp Pathol 7: 9050-9055, 2014

5. Liang N, Xie J, Liu F, Xu D, Yu X, Tian Y, Song M and Zhang J: Male breast metastases from nasopharyngeal carcinoma: A case report and literature review. Oncol Lett 7: 1586-1588, 2014.

6. Lee AW, Ngan RK, Tung SY, Cheng A, Kwong DL, Lu TX, Chan AT, Chan LL, Yiu H, Ng WT, et al: Preliminary results of trial NPC-0501 evaluating the therapeutic gain by changing from concurrent-adjuvant to induction-concurrent chemoradiotherapy, changing from fluorouracil to capecitabine, and changing from conventional to accelerated radiotherapy fractionation in patients with locoregionally advanced nasopharyngeal carcinoma. Cancer 121: 1328-1338, 2015.

7. Khalil EM and Anwar MM: Treatment results of pediatric nasopharyngeal carcinoma, NCI, Cairo University experience. J Egypt Natl Canc Inst 27: 119-128, 2015.

8. Wang Y, Lan G, Si Y, Deng Z, Sun J, Yang Y, Han X, Weng J and Zhou F: Treatment and outcome of recurrent cervical lymph nodes in patients with nasopharyngeal carcinoma after radiotherapy. Zhonghua Er Bi Yan Hou Tou Jing Wai Ke Za Zhi 51: 183-188, 2016 (In Chinese).

9. Casanova M, Ozyar E, Patte C, Orbach D, Ferrari A, Veyrat-Follet C, Errihani H, Pan J, Zhang L, Shen L, et al: International randomized phase 2 study on the addition of docetaxel to the combination of cisplatin and 5-fluorouracil in the induction treatment for nasopharyngeal carcinoma in children and adolescents. Cancer Chemother Pharmacol 77: 289-298, 2016.

10. Monteiro Gil O, Oliveira NG, Rodrigues AS, Laires A, Ferreira TC, Limbert E and Rueff J: Possible transient adaptive response to mitomycin $\mathrm{C}$ in peripheral lymphocytes from thyroid cancer patients after iodine-131 therapy. Int J Cancer 102: 556-561, 2002.

11. Stajduhar KI, Neithercut J, Chu E, Pham P, Rohde J, Sicotte A and Young K: Thyroid cancer: Patients' experiences of receiving iodine-131 therapy. Oncol Nurs Forum 27: 1213-1218, 2000.

12. Risse JH, Menzel C, Grunwald F, Strunk H, Biersack HJ and Palmedo H: Therapy of hepatocellular cancer with iodine-131-lipiodol. Rom J Gastroenterol 13: 119-124, 2004. 
13. Zhao YS, Yang HL and Liu CZ: Inhibitory effects of immunotargeting of Chinese cobra cytotoxin and iodine-131 against nasopharyngeal carcinoma cells in vitro. Nan Fang Yi Ke Da Xue Xue Bao 28: 1235-1236, 2008 (In Chinese).

14. Song JJ, Lin YS, Zhu L and Li F: Efficacy of iodine-131 in treating hyperthyroid heart disease. Zhongguo yi xue ke xue yuan xue bao 35: 166-170, 2013 (In Chinese)

15. Gultekin SS and Sahmaran T: The efficacy of patient-dependent practices on exposure rate in patients undergoing iodine-131 ablation. Health Phys 104: 454-458, 2013.

16. Zhu C, Ma X, Hu Y, Guo L, Chen B, Shen K and Xiao Y: Safety and efficacy profile of lenvatinib in cancer therapy: A systematic review and meta-analysis. Oncotarget 7: 44544-44557, 2016.

17. Hutson TE: Targeted therapies for the treatment of metastatic renal cell carcinoma: Clinical evidence. Oncologist 2 (Suppl 16): S14-S22, 2011

18. Matsui J and Funahashi Y: Preclinical biomarker research and patient stratification of molecular target agents: The anti-angiogenic inhibitor lenvatinib mesylate (E7080). Nihon Yakurigaku Zasshi 142: 162-166, 2013 (In Japanese).

19. Strazzulla A, Barreca GS, Giancotti A, Pisani V, Costa C Zicca E, La Boria A, Roveda L, Liberto MC, Tucci L, et al: Nasopharyngeal carcinoma: Review of the literature with a focus on therapeutical implications. Infez Med 23: 224-229, 2015.

20. Kuznar W: Lenvatinib extends survival in metastatic renal-cell carcinoma. Am Health Drug Benefits 8: 18, 2015.

21. May D, Itin A, Gal O, Kalinski H, Feinstein E and Keshet E: Ero1-L alpha plays a key role in a HIF-1-mediated pathway to improve disulfide bond formation and VEGF secretion unde hypoxia: Implication for cancer. Oncogene 24: 1011-1020, 2005.

22. Spano JP, Milano G and Baselga J: EGFR/VEGF signalling pathway in colorectal cancer: The way we are! Bull Cancer 92: S3-S4, 2005 (In English, French).

23. Molina AM, Hutson TE, Larkin J, Gold AM, Wood K, Carter D, Motzer R and Michaelson MD: A phase 1b clinical trial of the multi-targeted tyrosine kinase inhibitor lenvatinib (E7080) in combination with everolimus for treatment of metastatic renal cell carcinoma (RCC). Cancer Chemother Pharmacol 73 181-189, 2014.

24. Soto-Nunez M, Diaz-Morales KA, Cuautle-Rodriguez P, Torres-Flores V, López-González JS, Mandoki-Weitzner JJ and Molina-Guarneros JA: Single-cell microinjection assay indicates that 7-hydroxycoumarin induces rapid activation of caspase-3 in A549 cancer cells. Exp Ther Med 10: 1789-1795, 2015.

25. Dunmade AD and Ademola-Popoola DS: Neuro-ophthalmic manifestation of nasopharyngeal carcinoma at Ilorin: A five year review. Niger J Clin Pract 11: 376-378, 2008.

26. Hui D, Elsayem A, Li Z, De La Cruz M, Palmer JL and Bruera E: Antineoplastic therapy use in patients with advanced cancer admitted to an acute palliative care unit at a comprehensive cancer center: A simultaneous care model. Cancer 116: 2036-2043, 2010.

27. Beck JT: Potential role for mammalian target of rapamycin inhibitors as first-line therapy in hormone receptor-positive advanced breast cancer. OncoTargets Ther 8: 3629-3638, 2015.

28. Okamoto K, Kodama K, Takase K, Sugi NH, Yamamoto Y, Iwata $\mathrm{M}$ and Tsuruoka $\mathrm{A}$ : Antitumor activities of the targeted multi-tyrosine kinase inhibitor lenvatinib (E7080) against RET gene fusion-driven tumor models. Cancer Lett 340: 97-103, 2013

29. Yokokura S, Yurimoto S, Matsuoka A, Imataki O, Dobashi H, Bandoh S and Matsunaga T: Calmodulin antagonists induce cell cycle arrest and apoptosis in vitro and inhibit tumor growth in vivo in human multiple myeloma. BMC Cancer 14: 882, 2014.

30. Bahrambeigi V, Ahmadi N, Moisyadi S, Urschitz J, Salehi R and Haghjooy Javanmard S: PhiC31/PiggyBac modified stromal stem cells: Effect of interferon gamma and/or tumor necrosis factor (TNF)-related apoptosis-inducing ligand (TRAIL) on murine melanoma. Mol Cancer 13: 255, 2014.
31. Supriatno, Yuletnawati SE and Widiasto A: Effect of intratumoral injection of mutant type p27Kip1 followed by in vivo electroporation on radiotherapy-resistant human oral tongue cancer xenografts. Mol Med Rep 4: 41-46, 2011.

32. Howard DM, Kearfott KJ, Wilderman SJ and Dewaraja YK: Comparison of I-131 radioimmunotherapy tumor dosimetry: Unit density sphere model versus patient-specific Monte Carlo calculations. Cancer Biother Radiopharm 26: 615-621, 2011.

33. Castellani MR, Aktolun C, Buzzoni R, Seregni E, Chiesa C, Maccauro M, Aliberti GL, Vellani C, Lorenzoni A and Bombardieri E: Iodine-131 metaiodobenzylguanidine (I-131 MIBG) diagnosis and therapy of pheochromocytoma and paraganglioma: Current problems, critical issues and presentation of a sample case. Q J Nucl Med Mol Imaging 57: 146-152, 2013.

34. Harisankar CN, Mittal BR, Bhattacharya A, Kashyap R and Bhansali A: Iodine-131 meta-iodobezylguanidine single photon emission computed tomography/computerized tomography in diagnosis of neuro-endocrine tumors. Indian J Nucl Med 27: $55-58,2012$.

35. Shao H, Zhang J, Sun Z, Chen F, Dai X, Li Y, Ni Y and Xu K: Necrosis targeted radiotherapy with iodine-131-labeled hypericin to improve anticancer efficacy of vascular disrupting treatment in rabbit VX2 tumor models. Oncotarget 6: 14247-14259, 2015.

36. Shumaker RC, Zhou M, Ren M, Fan J, Martinez G, Aluri J and Darpo B: Effect of lenvatinib (E7080) on the QTc interval: Results from a thorough QT study in healthy volunteers. Cancer Chemother Pharmacol 73: 1109-1117, 2014

37. Boss DS, Glen H, Beijnen JH, Keesen M, Morrison R, Tait B, Copalu W, Mazur A, Wanders J, O'Brien JP, et al: A phase I study of E7080, a multitargeted tyrosine kinase inhibitor, in patients with advanced solid tumours. Br J Cancer 106: 1598-1604, 2012

38. Dubbelman AC, Rosing H, Nijenhuis C, Huitema AD, Mergui-Roelvink M, Gupta A, Verbel D, Thompson G, Shumaker R, Schellens JH and Beijnen JH: Pharmacokinetics and excretion of (14)C-lenvatinib in patients with advanced solid tumors or lymphomas. Invest New Drugs 33: 233-240, 2015.

39. Tohyama O, Matsui J, Kodama K, Hata-Sugi N, Kimura T, Okamoto K, Minoshima Y, Iwata M and Funahashi Y: Antitumor activity of lenvatinib (e7080): An angiogenesis inhibitor that targets multiple receptor tyrosine kinases in preclinical human thyroid cancer models. J Thyroid Res 2014: 638747, 2014.

40. Wang Y and Teng JS: Increased multi-drug resistance and reduced apoptosis in osteosarcoma side population cells are crucial factors for tumor recurrence. Exp Ther Med 12: 81-86, 2016.

41. Cao S, Wang T, Yan B, Lu Y, Zhao Y and Zhang S: Brain death is associated with endoplasmic reticulum stress and apoptosis in rat liver. Transplant Proc 46: 3297-3302, 2014.

42. Yang D, Gao L, Wang T, Qiao Z, Liang Y and Zhang P: Hypoxia triggers endothelial endoplasmic reticulum stress and apoptosis via induction of VLDL receptor. FEBS Lett 588: 4448-4456, 2014.

43. Ghosh S, Adhikary A, Chakraborty S, Bhattacharjee P, Mazumder M, Putatunda S, Gorain M, Chakraborty A, Kundu GC, Das T and Sen PC: Cross-talk between endoplasmic reticulum (ER) stress and the MEK/ERK pathway potentiates apoptosis in human triple negative breast carcinoma cells: Role of a dihydropyrimidone, nifetepimine. J Biol Chem 290: 3936-3949, 2015.

This work is licensed under a Creative Commons Attribution-NonCommercial-NoDerivatives 4.0 International (CC BY-NC-ND 4.0) License. 